\title{
ATTACHMENT STYLE AS A PREDICTOR OF RISKY SEXUAL BEHAVIOR IN ADOLESCENTS
}

\author{
${ }^{1}$ Amber Paulk and ${ }^{2}$ Ryan Zayac \\ ${ }^{1}$ Department of Human Environmental Sciences and Sociology and Family Studies, \\ ${ }^{2}$ Department of Psychology, \\ University of North Alabama, Florence, AL, USA
}

Received 2013-06-04; Revised 2013-06-19; Accepted 2013-06-21

\begin{abstract}
The aim of the current study was to examine attachment style as a predictor of risky sexual behavior in a younger, more vulnerable sample than previously investigated in the literature: High school aged adolescents. The hypothesized associations among the variables were partially supported. Contrary to our predictions, there was no significant association between avoidance and risky sexual behavior. However, higher anxiety was positively associated with risky sexual behavior. Finally, the prediction that the highest levels of risky sexual behavior would be found for those individuals with higher levels of anxiety and higher levels of avoidance (i.e., insecure attachment style) was supported.
\end{abstract}

Keywords: Risky Sexual Behavior, Attachment Style, Adolescents

\section{INTRODUCTION}

Developed by Bowlby (1982; 1983), attachment theory asserts that individuals from internal working models, or mental representations of self and other, which impact their feelings about and behavior in, relationships. Models of self can be either positive (i.e., one feels worthy of love and care) or negative (i.e., one feels unworthy of love and care) and models of other can be either positive (i.e., one feels others are available and responsive) or negative (i.e., one feels others are distant or rejecting) (Batholomew, 1990). A secure attachment style is characterized by possessing both positive models of self and of other. In contrast, insecure attachment styles are categorized as anxious or avoidant. An anxious attachment style is characterized by a negative model of self, while an avoidant attachment style is characterized as a negative model of other (Feeney and Noller, 1996). Although attachment was initially studied in the context of parent-child relationships, Bowlby (1982) contended that subsequent interactions with other relationship partners could potentially update one's working models. In subsequent research, attachment was studied in other types of relationships (e.g., with peers and romantic partners) and these studies found that models of self and other can vary across different types of relationships (Collins and Read, 1994; Guardia et al., 2000).

One of the primary differences between romantic relationship attachment and other types of attachment relationships (e.g., peers, parents) is that romantic relationships include the integration of sexual behavior (Bowlby, 1982; 1983; Shaver et al., 1988). Indeed, studies have shown that couples report a reciprocal relationship between attachment and their sexual relationships (Feeney, 1999; Sprecher and Cate, 2004). A series of reviews by Feeney (1999) and Feeney and Noller (2004) indicate that attachment styles influence how adults interpret many aspects of their romantic relationships, including their sexual attitudes and beliefs and several studies have focused on the association between romantic relationship attachment and sexual behavior (Bogaert and Sadava, 2002; Gentzler and Kerns, 2004; Strachman and Impett, 2009).

Corresponding Author:Amber Paulk, Department of Human Environmental Sciences and Sociology and Family Studies,

University of North Alabama, Florence, AL, USA 
In a study of college undergraduates, Strachman and Impett (2009) examined how attachment style (i.e., avoidance or anxiety) was associated with condom use across a period of 14 days. Attachment style was measured using the Experiences in Close Relationships Scale (Brennan et al., 1998), which is a 36 item self-report measure of attachment style which yields scores for anxiety and avoidance, with low scores on both indicating a secure attachment style. During the two week period, participants were asked to keep a diary of their sexual contact and indicate if a condom was used during intercourse. The study found that participants with an anxious attachment style were less likely to use condoms. The association between attachment style and condom use remained significant when controlling for other related variables such as gender, alternative birth control use, sex frequency and knowledge of a partner's sexual history.

In a similar study, Gentzler and Kerns (2004) examined the association between attachment style and sexual behaviors in college students. Attachment was measured using the Experiences in Close Relationships Scale (Brennan et al., 1998) and sexual behavior was measured through a detailed sexual history. The study found that avoidant and anxious attachment styles were associated with negative sexual experiences. Most notably, participants identified as anxious or avoidant in their attachment style were significantly more likely than those with a secure attachment style to report participating in unwanted, although not forced, sexual experiences.

Bogaert and Sadava (2002) examined the association between attachment style and a variety of sexual behaviors (e.g., number of sexual partners, age of first sexual experience, frequency of sexual behaviors in the past year, whether an affair had occurred in the past year and consistent condom usage) in young adults. Attachment style was measured using the Experiences in Close Relationships Scale (Brennan et al., 1998) and sexual behavior was measured through detailed sexual history. The study found that participants with an anxious attachment style typically had an early first intercourse, more lifetime partners, more infidelity and took fewer sexual precautions (e.g., condom use).

Feeney et al. (2000) measured college students' attachment, sexual behavior and perceived riskiness of that behavior. Anxiety was associated with drug use behavior during sexual contact, unsafe sex and negative attitudes toward condoms; while avoidance was associated with a more cautious approach to sexual behavior. In a similar study, Schachner and Shaver (2004) examined the association between attachment style and motives for having sex. They found that participants with an anxious attachment style reported having sex to decrease insecurity and increase closeness with their partners, while participants with an avoidant attachment style reported having sex to impress their friends, especially if they were having sex casually with uncommitted partners.

\subsection{Study Problem and Hypotheses}

The aforementioned studies contributed to the understanding of the association between attachment styles and sexual behavior in a sample older (i.e., college-aged) participants. The purpose of the current study was to examine attachment styles as a predictor of risky sexual behavior in a younger, more vulnerable sample: high school-aged adolescents. It was predicted that adolescents scoring high on measures of anxiety would have more risky sex in order to decrease relationship insecurity and promote intimacy with their partners. It was predicted that adolescents scoring high on measures of avoidance would be less likely to engage in risky sexual behavior.

Specifically, it was hypothesized that there would be a significant, positive association between anxiety and risky sexual behavior and a significant, negative association between avoidance and risky sexual behavior. Previous research, such as Anders and Tucker (2000), suggests that perhaps the most insecure individuals are those score both high in anxiety and high in avoidance. It is for this reason that the authors also hypothesized that the interaction between anxiety and avoidance would make a unique, significant contribution to risky sexual behavior and that the highest levels of risky sexual behavior would be found for those individuals with higher levels of anxiety and higher levels of avoidance.

\section{MATERIALS AND METHODS}

\subsection{Procedure}

Participants were recruited from Family and Consumer Sciences (FCS) courses where teachers delivered a curriculum (Relationship Smarts) in regularly scheduled classes and administered a pre-test and posttest to their students. Teachers in FCS courses received training in the procedures of data collection at a centralized training session. Both student assent and 
parental consent for student participation in the research were obtained with the cooperation of participating teachers. The surveys were administered during regularly scheduled classes.

\subsection{Participants}

The data were collected from 258 adolescents attending grades 9-12 in a public high school in the Pacific Northwest. On average, participants were 16.6 years old $(\mathrm{SD}=0.984)$ and in the 10th grade. Approximately half of the sample was female (47.5\%) and half was male $(52.5 \%)$. In terms of race/ethnicity, $90 \%$ of participants were Caucasian, $6 \%$ were AfricanAmerican and the remaining 4\% included Hispanic, Native American and Asian American students. This racial composition approximates well the racial makeup of the participating high school.

For the purposes of the current study, sexual intercourse was defined as a "male's penis inside a female's vagina."Approximately fifty percent of the sample $(\mathrm{N}=129)$ reported they had previously engaged in sexual intercourse, while the other fifty percent reported they had never had sexual intercourse $(\mathrm{N}=126)$. Of the participants who had engaged in sexual intercourse, the average age of first intercourse was 13.01 years old $(\mathrm{SD}=3.12)$ and the average number of sexual partners was $3(\mathrm{SD}=1.70)$. When engaging in sexual intercourse, $50.4 \%$ of participants reported that they used condoms "all of the time," $22.8 \%$ reported they used condoms "most of the time," $8.7 \%$ reported they used condoms "about half of the time," $7.1 \%$ reported they used condoms "less than half of the time," and $11.0 \%$ reported they used condoms "none of the time."

\subsection{Measures}

\subsubsection{Attachment of Assessment Style}

The Experiences in Close Relationships Scale (ECRS) (Brennan et al., 1998) was used to assess adolescents' attachment. The ECRS contains 36 items. Eighteen ECRS items (i.e., nine for each subscale) were selected for use in the current investigation. The nine items for each subscale were chosen after conducting an Exploratory Factor Analysis (EFA) with a convenient sample of 294 college students. An EFA with the 18 original avoidance items found three factors and an alpha of 0.93. A second EFA was conducted with the 9 selected avoidance items and found one factor and an alpha of 0.89. Analyses revealed that those nine avoidance items captured $93 \%$ of the variance of the original 18 items; therefore, those nine items were used in the current investigation. Another EFA was conducted for the anxiety subscale. An EFA with the 18 original anxiety items found three factors and an alpha of 0.91. The second EFA was conducted with the 9 selected anxiety items and found two factors and an alpha of 0.86 . Those nine anxiety items captured $93 \%$ of the variance of the original 18 items; therefore, those items were used in the current investigation.

On the ECRS, participants are asked to rate the degree which they agree/disagree with each item on a scale of 1 to 5 , ' 1 ' being "strongly disagree" and ' 5 ' being "strongly agree." Nine of the items are designed to tap into participants' level of anxiety in romantic relationships. An example of an anxiety item is: "I often wish that my partners' feelings for me were as significant as my feelings for him/her." Nine of the items are designed to tap into participants' level of avoidance in romantic relationships. An example of an avoidance item is: "I am nervous when romantic partners get too close to me." Responses to anxious and avoidant items were summed and averaged to obtain participants' means across each dimension, with higher scores indicating higher levels of anxiety and avoidance. In the current sample, the reliabilities for the two observed composites were 0.63 for avoidance and 0.82 for anxiety.

\subsection{Assessment of Risky Sexual Behavior}

All participants were asked to complete several questions about their sexual behavior. First, participants were asked "Have you ever had sexual intercourse? 'Sexual intercourse' means having sex with the male's penis inside the female's vagina. This is sometimes called 'going all the way." Participants who had engaged in sexual intercourse were asked a series of questions to assess the risk in their sexual behavior. First, participants were asked "How old were you when you first had sexual intercourse?" with the response options of 9 years old or younger, 10, 11, 12, $13,14,15,16$ years old or older, or I have never had sexual intercourse. Next, participants were asked "During your life, with how many different people have you had sexual intercourse?" with the response options of 1 person, 2, 3, 4, 5 people or more, or I have never 
had sexual intercourse. Finally, participants were asked "In the last month, how much of the time did you or your sexual partner use a condom when you had sexual intercourse?" with the response options of none of the times, less than half of the times, about half of the times, most of the times, all of the times, or I have never had sexual intercourse.

Other studies have found it appropriate to combine several questions to create a composite score to represent risky sexual behavior (Bachanas et al., 2002; Kogan et al., 2010; Timmermans et al., 2008). For the purposes of this study, these three items were standardized and combined to create an aggregate variable to represent risky sexual behavior. First, the response options for the risky sexual behavior questions were re-coded into one of six categories: no risk, least risky, somewhat risky, moderately risky, quite risky and mostly risky. The age of first sex responses were re-coded as follows: $0=$ no risk/never had sex; 1 = least risky/16 years old or older; $2=$ somewhat risky/14-15 years old; $3=$ moderately risky/12-13 years old; 4 = quite risky/10-11 years old; $5=$ most risky $/ 9$ years or younger. The number of sexual partners responses were re-coded as follows: 0 $=$ no $\mathrm{risk} /$ never had sex; $1=$ least $\mathrm{risky} / 1$ person; $2=$ somewhat risky $/ 2$ people; $3=$ moderately risky $/ 3$ people; $4=$ quite risky $/ 4$ people; $5=$ most risky $/ 5$ people or more. The condom use responses were recoded as follows: $0=$ no risk $/$ never had sex; $1=$ least risky/all of the time; $2=$ somewhat risky/most of the time; 3 = moderately risky/about half of the time; $4=$ quite risky/less than half of the time; $5=$ most risky/none of the time. Once all risky sexual behavior questions were re-coded they were combined and averaged to create a single item "Risky Sexual Behavior." The reliability for the three items was 0.79 .

\section{RESULTS}

A descriptive analysis was conducted to make sure that all values were in range and to describe central tendencies and variance of the measures. Items were recoded for contributing in the correct direction of composite score. Then, aggregated scales were constructed. Reliability of each scale was tested to ensure the quality of the data. Table 1 for the alphas, means, standard deviation, minimum values, maximum values, skewness and kurtosis of each aggregated scale and subscale. Correlations were calculated for all major constructs and are presented in Table 2.

First, the main effects hypotheses were tested. A linear regression analysis revealed that avoidance and anxiety were able to explain $11 \%$ of the variance in risky sexual behavior [Adj. $\mathrm{R}^{\wedge}=0.106, \mathrm{~F}(2,25)=1.40, \mathrm{p}<0.05$ ]. The hypothesized associations among the variables were tested and partially supported. A higher anxiety score $(\mathrm{t}$ $=1.66, \beta=-0.117, \mathrm{p}<0.05)$ was associated with a higher risky sexual behavior score. However, there was no significant association between avoidance and risky sexual behavior score $(t=-0.856, \beta=-0.060, p=0.393)$.

The next step in the analyses was to test our interaction hypothesis. It was predicted that the interaction between anxiety and avoidance would make a unique, significant contribution to risky sexual behavior after controlling for the main effects of anxiety and avoidance. Kline and Dunn (2000) suggest that all multiplicative interaction terms be created from centered variables in order to reduce collinearity between the main effect variables and their interaction terms. Therefore, the anxiety and avoidance variables were centered by subtracting the mean from each value before using it to create the interaction term (avoidance*anxiety).

Table 1. Descriptive statistics (alphas, means, standard deviations, range, skewness and kurtosis) for all aggregate scales

\begin{tabular}{lccccrr}
\hline & Cronbach & & & & \\
Variable & Alpha & Mean & SD & Range & Skewness & Kurtosis \\
\hline Anxiety & 0.820 & 2.63 & 0.829 & $1-5$ & 0.096 & -0.033 \\
Avoidance & 0.627 & 2.52 & 0.621 & $1-5$ & -0.315 & -0.315 \\
Risky sex composite & 0.788 & 1.48 & 1.42 & $1-5$ & 0.226 & -1.380 \\
\hline
\end{tabular}

Table 2. Correlations for aggregate scales

\begin{tabular}{llllll}
\hline & 1 & 2 & 3 & 4 & 5 \\
\hline 1. Anxiety & 1.000 & & & & \\
2. Avoidance & $0.428^{* * *}$ & 1.000 & & & \\
3. Age of first sex & 0.067 & $0.118^{*}$ & 1.000 & & \\
4. Number of partners & $-0.115^{*}$ & 0.037 & $0.560^{* *}$ & 1.000 & 1.000 \\
5. Condom use & 0.043 & 0.082 & $0.585^{* *}$ & $0.550^{* *}$ & $0.820^{* *}$ \\
6. Risky sex composite & -0.010 & 0.091 & $0.846^{* *}$ & $0.861^{* *}$ & 1 \\
\hline
\end{tabular}

$* \mathrm{p}<0.05, * * \mathrm{p}<0.01, * * * \mathrm{p}<0.001$ 
The interaction term (avoidance*anxiety) was added to the model. A linear regression analysis revealed that when the interaction term was added, the model was able to explain $16 \%$ of the variance in risky sexual behavior [Adj. $\mathrm{R}^{\wedge}=0.159, \mathrm{~F}(2,25)=2.10, \mathrm{p}<0.05$ ]. The hypothesized association was supported. Higher interaction (avoidance*anxiety) scores were associated with higher levels of risky sexual behavior $(\mathrm{t}=-1.85$, $\beta=-0.117, \mathrm{p}<0.05)$.

\section{DISCUSSION}

The central goal of the current investigation was to examine the association between romantic relationship attachment style and risky sexual behavior in adolescents. It was predicted that: (a) anxiety would be positively related to risky sexual behavior, (b) avoidance would be negatively associated with risky sexual behavior, (c), that the interaction between anxiety and avoidance would make a unique, significant contribution to risky sexual behavior and (d) that the highest levels of risky sexual behavior would be found for those individuals with higher levels of anxiety and higher levels of avoidance (i.e., insecure style). These hypotheses were partially supported.

A higher anxiety score was positively associated with risky sexual behavior. In order to understand this finding, it is important to remember that high anxiety maps onto a negative model of self, which is defined as a preoccupation with seeking closeness, love and approval from others (Batholomew, 1990; Hazan and Shaver, 1987). Since anxious adolescents are preoccupied with seeking out closeness with others, it is not surprising that they would seek to please others through engaging in sexual behaviors, particularly those that are risky (e.g., early sexual initiation, multiple sexual partners, inconsistent condom use).

Contrary to our predictions, there was no significant association between avoidance and risky sexual behavior. In order to interpret why the association between avoidance and risky sexual behavior was insignificant, it is important to explore the common characteristics of avoidant individuals. It is important to note that high avoidance maps onto a negative model of other, which is defined as a discomfort with close emotional relationships, an important need to feel independent and preference to not depend on others (Feeney and Noller, 1996). It would theoretically follow that individuals who use an avoidant style may lack the trust in others necessary to engage in relationships, which may impact their opportunities to engage in any form of sexual behavior. Therefore, the authors speculate that avoidant individuals may be avoiding relationships and closeness, which includes engagement in sexual activities. So, the reason that avoidance is not associated with risky sexual behavior is because avoidant individuals are engaging in less sexual behavior altogether. However, more research is necessary to understand if avoidance-adolescents are more likely than others to avoid sexual situations.

Finally, the prediction that the highest levels of risky sexual behavior would be found for those individuals with higher levels of anxiety and higher levels of avoidance (i.e., insecure style) was supported. The results of the interaction model were consistent with romantic relationship attachment theory (Batholomew, 1990). When attachment anxiety was low, the lowest engagement in risky sexual behavior was seen when avoidance was also low. Therefore, adolescents who had secure models of self and other were the least likely to engage in risky sexual behavior. The opposite was also true. Adolescents who were high in avoidance and anxiety were the most likely to engage in risky sexual behavior.

There were several limitations in the current investigation. The current study only consisted of crosssectional data. Adolescents were only surveyed at one point in time, which makes it impossible to evaluate change and the direction of effects. Therefore, the results are simply correlational and causality cannot be established. Therefore, longitudinal research is necessary in order to establish the directional assumptions presented in the study. Another limitation in the current investigation was that it lacked sufficient power to conduct analyses among all represented ethnic groups. Future studies would benefit from attempting to recruit more diverse populations and investigating if the associations among attachment style and risky sexual behavior differ cross-culturally.

\section{CONCLUSION}

The introduction of romantic relationships during adolescence means that teens must begin to make critical choices regarding sexual behavior. Therefore, it is critical to understand the processes that underlie teens' sexual decision-making. The current study sought to understand adolescents' engagement in risky sexual behavior from a romantic relationship attachment style perspective. The findings of the current study indicate that adolescents with secure models of self and other are the least likely to engage in risky sexual behavior. However, adolescents who are anxious and those who are high in avoidance and anxiety are the most likely to engage in risky sexual behavior. Therefore, it is critical that these teens are identified as the most at-risk and be targeted by sexual health education programming. 


\section{REFERENCES}

Anders, S.L. and J.S. Tucker, 2000. Adult attachment style, interpersonal communication competence and social support. Personal Relat., 7: 379-389. DOI: 10.1111/j.1475-6811.2000.tb00023.x

Bachanas, P.J., M.M. Morris, J.K. Lewis-Gess, E.J. SarettCuasay and K. Sirl et al., 2002. Predictors of risky sexual behavior in African American adolescent girls: Implications for prevention interventions. J. Pediatr. Psychol., 27: 519-530. DOI: 10.1093/jpepsy/27.6.519

Batholomew, K., 1990. Avoidance of intimacy: An attachment perspective. J. Soc. Personal Relat., 7: 147-178. DOI: $10.1177 / 0265407590072001$

Bogaert, A.F. and S. Sadava, 2002. Adult attachment and sexual behavior. Personal Relat., 9: 191-204. DOI: 10.1111/1475-6811.00012

Bowlby, J., 1982. Attachment and loss: Retrospect and prospect. Am. J. Orthopsychiatry, 52: 664-678. DOI: 10.1111/j.1939-0025.1982.tb01456.x

Bowlby, J., 1983. Attachment: Attachment and Loss. 2nd Edn., Basic Books, New York, ISBN-10: 0465005438, pp: 436.

Brennan, K., C. Clark and P. Shaver, 1998. Self-Report Measure of Adult Attachment: An Integrative Overview. In: Attachment Theory and Close Relationships, Simpson, J.A. and W.S. Rholes (Eds.), Guilford Press, New York, ISBN-10: 1572301023, pp: 46-76.

Collins, N. and S. Read, 1994. Cognitive Representations of Attachment: The Structure and Function of Working Models. In: Attachment Processes in Adulthood, Bartholomew, K. and D. Perlman (Eds.), Jessica Kingsley Publishers, London, ISBN-10: 1853021725, pp: 53-90.

Feeney, J. and P. Noller, 1996. Adult attachment. Sage, Thousand Oaks, ISBN-10: 0803972237, pp: 176.

Feeney, J. and P. Noller, 2004. Attachment and Sexuality in Close Relationships. In: The Handbook of Sexuality in Close Relationships, Harvey, J.H., A. Wenzel and S. Sprecher (Eds.), Routledge, ISBN10: 0805845488, pp: 183-201.

Feeney, J.A., 1999. Issues of closeness and distance in dating relationships: Effects of sex and attachment style. J. Soc. Personal Relat., 16: 571-590. DOI: 10.1177/0265407599165002

Feeney, J.A., C. Peterson, C. Gallois and D.J. Terry, 2000. Attachment style as a piedictor of sexual attitudes and behavior in late adolescence. Psychol. Health, 14: 1105-1122. DOI: 10.1080/08870440008407370
Gentzler, A.L. and K.A. Kerns, 2004. Associations between insecure attachment and sexual experiences. Personal Relat., 11: 249-265. DOI: 10.1111/j.1475-6811.2004.00081.x

Guardia, J.G.L., R.M. Ryan, C.E. Couchman and E.L. Deci, 2000. Within-person variation in security of attachment: A self-determination theory perspective on attachment, need fulfillment and well-being. J. Personality Soc. Psychol., 79: 367-384. PMID: 10981840

Hazan, C. and P. Shaver, 1987. Romantic love conceptualized as an attachment process. J. Personality Soc. Psychol., 52: 511-524. DOI: 10.1037/0022-3514.52.3.511

Kline, T.J.B. and B. Dunn, 2000. Analysis of interaction terms in structural equation models: A non-technical demonstration using the deviation score approach. Canadian J. Behav. Sci., 32: 127-132. DOI: 10.1037/h0087107

Kogan, S.M., S.R.H. Beach, R.A. Philibert, G.H. Brody and Y.F. Chen et al., 2010. 5-HTTLPR status moderates the effect of early adolescent substance use on risky sexual behavior. Health Psychol., 29: 471-476. DOI: 10.1037/a0020594

Schachner, D.A. and P.R. Shaver, 2004. Attachment dimensions and sexual motives. Personal Relat., 11: 179-195. DOI: $10.1111 / j .1475-6811.2004 .00077 . x$

Shaver, P., C. Hazan and D. Bradshaw, 1988. Love as Attachment. In: The Psychology of Love Sternberg, R.J. and M.L. Barnes (Eds.), Yale University Press, Hew Haven, CT., ISBN-10: 0300045891, pp: 68-99.

Sprecher, S. and R. Cate, 2004. Sexual Satisfaction and Sexual Expression as Predictors of Relationship Satisfaction and Stability. In: The Handbook of Sexuality in Close Relationships, Harvey, J.H., A. Wenzel and S. Sprecher (Eds.), Routledge, ISBN10: 0805845488, pp: 235-256.

Strachman, A. and E.A. Impett, 2009. Attachment orientations and daily condom use in dating relationships. J. Sex Res., 4: 319-329. DOI: 10.1080/00224490802691801

Timmermans, M., P.A.C.V. Lier and H.M. Koot, 2008. Which forms of child/adolescent externalizing behaviors account for late adolescent risky sexual behavior and substance use? J. Child Psychol. Psychiatry, 49: 386-394. DOI: 10.1111/j.14697610.2007.01842.x 